\title{
The Role of Magnetic Resonance Imaging in the Evaluation of Trigeminal Neuralgia
}

\section{VIJAYKUMAR KENCHANAHALLI RANGASWAMY, MASKAL REVANNA SRINIVAS, DEEPASHRI BASAVALINGU, BANGALORE RANGASWAMY NAGARAJ}

\begin{abstract}
Introducation: Trigeminal neuralgia is characterized by electric shock-like pain, abrupt in onset and brief, in the distribution of one or more divisions of the trigeminal nerve. Trigeminal neuralgia can occur due to lesions affecting trigeminal nerve, either vascular, neoplastic, infectious or inflammatory. Neurovascular compression is found to be the cause in most cases.

Aim: The aim of this study is to study and classify brain magnetic resonance imaging findings in patients aged $>18$ years who presented with clinical symptoms of trigeminal neuralgia.
\end{abstract}

Materials and Methods: Clinical records and imaging studies of 75 patients between the ages of 18-60 years who presented to the Dept of Radio-diagnosis, BMCRI for brain magnetic resonance imaging during May 2014 to May 2015 were analyzed retrospectively.

Results: Neurovascular compression at the root entry zone was found to be the most common cause of trigeminal neuralgia in our study. Some of the other causes that were identified in our study include cerebellopontine (CP) angle tumors, brain stem infarct, multiple sclerosis (MS), etc.

Conclusion: The role of magnetic resonance imaging in the evaluation of trigeminal neuralgia is to identify those causes that are amenable to surgical treatment such as neurovascular compression and tumors.

\section{INTRODUCTION}

Trigeminal nerve is the fifth and largest cranial nerve and arises from one motor nucleus and three sensory nuclei in the brain stem. The three sensor nuclei, namely- a. the mesencephalic nucleus, supplying proprioceptive fibres to the muscles of face, mastication, tongue and orbit; $b$. the main sensory nucleus, providing touch fibers to all three divisions of trigeminal nerve and c. the spinal nucleus, responsible for pain and temperature, are arranged in a column that extends from the mid brain to the upper cervical spinal cord. The motor nucleus of trigeminal nerve is present in the upper pons and controls the muscles of mastication [1].

The root entry zone (REZ) of trigeminal nerve is its segment in the prepontine cistern where it is most susceptible to neurovascular conflict and signifies the transition from central to peripheral myelin. Trigeminal nerve forms Gasserian ganglion in a CSF filled space in the petrous apex called Meckel's cave from which, ophthalmic (V1), maxillary (V2) and mandibular (V3) branches of trigeminal nerve arise [1,2] [Table/Fig-1].

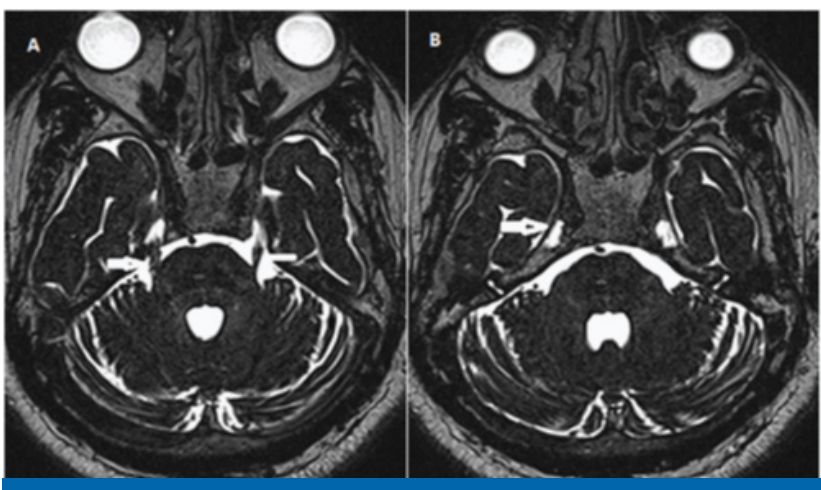

[Table/Fig-1]: Normal appearance of trigeminal nerve on CISS sequence. A: Trigeminal nerves in the pre- pontine cistern in axial image(yellow arrows). Pons- is denoted by *; B: Bilateral Meckel's caves (yellow arrow) in a section caudal to the above image. Basilar artery is seen anterior to the pons.

Trigeminal neuralgia is characterized by electric shock-like pain, abrupt in onset and brief, in the distribution of one or more divisions of the trigeminal nerve. Trigeminal neuralgia can be classified into two types- classical and symptomatic 
by the criteria laid down by the International Headache Society. Hereafter, we will not distinguish the two, with 'trigeminal neuralgia' used for referring to both types [3].

Previous studies have reported the prevalence of trigeminal neuralgia as ranging between 4-13 per 1 lakh individuals. Highest incidence of trigeminal neuralgia is seen in the age group of 4060 years and shows slight female predominance $[4,5]$.

The causes of trigeminal neuralgia vary according to the site of involvement. At the level of trigeminal nucleus, the common causes include infarcts, cavernoma, demyelinating plaques of multiple sclerosis (MS), brain stem glioma, etc. At the level of prepontine cistern, the common causes of trigeminal neuralgia are neurovascular compression, aneurysms and cerebellopontine (CP) angle tumors. Other recognized causes of trigeminal neuralgia include base of skull tumors, sarcoid, pituitary macroadenoma and perineural spread of salivary gland tumors $[2,6]$. The most common cause of trigeminal neuralgia in previous studies was found to be neurovascular contact most commonly by the superior cerebellar artery and its branches [6]. Basilar artery and its branches are shown in [Table/Fig-2].

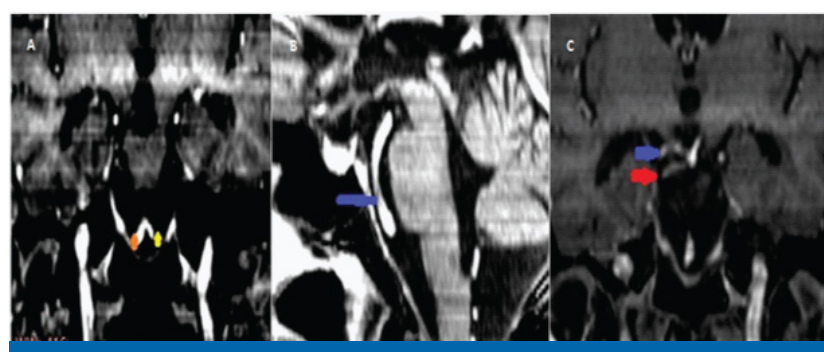

[Table/Fig-2]: Basilar artery: Course and branches. A and B: Coronal and sagittal contrast enhanced fat suppressed $\mathrm{T} 1$ weighted image showing bilateral vertebral arteries( orange and yellow marks) joining to form the basilar artery ( blue arrow); C: Coronal contrast enhanced fat suppressed T1 weighted image showing superior cerebellar artery and posterior cerebral artery (red and blue arrow respectively).

The purpose of imaging in trigeminal neuralgia is to identify the cause and to further identify those patients in whom surgical treatment is possible. The foremost imaging modality for this purpose is magnetic resonance imaging (MRI). Magnetic resonance imaging has changed the way we look at cranial nerves. The superior soft tissue resolution and newer sequences have made it possible to evaluate the trigeminal nerve from the level of its nuclei to its distal branches. One such sequence, the 3D CISS( constructive interference in steady state) sequence is a heavily T2 weighted isotropic sequence in which CSF appears bright and rest of the intracranial structures including nerves, vessels, grey and white matter appear dark [7-9].

Harsha $\mathrm{KJ}$ described the various causes of trigeminal neuralgia and their detection by magnetic resonsnce imaging [10]. Neetu $S$ et al., in their study of patients with symptomatic trigeminal neuralgia due to causes other than neurovascular compression reiterated the role of magnetic resonance imaging and in particular diffusion tensor imaging in the demonstration of microstructural changes [11].

No study has been done previously to determine the frequency of various causes of trigeminal neuralgia in south Indian population and the proportion of cases that can be treated surgically. The objective of the present study was to identify the prevalence of abnormal magnetic resonance imaging and its causes using routine and 3 dimensional constructive interference in steady state (3D CISS) MR imaging sequence in South Indian population with trigeminal neuralgia diagnosed clinically.

\section{MATERIALS AND METHODS}

This was a prospective cross sectional descriptive study, conducted on 75 consecutive patients who presented to the Department of Radiodiagnosis, Victoria Hospital, Bangalore Medical College and Research Institute, for Magnetic resonance imaging evaluation of trigeminal neuralgia between May 2014 and May 2015. Their clinical data and imaging findings were analyzed. The age of the patients ranged between 25 to 70 years (average age- 55 years. All patients had clinical symptoms lasting at least 6 months in duration before presenting for the imaging study. Patients with contra-indication to magnetic resonance imagingclaustrophobia, cochlear implant, pacemaker etc., were excluded from the study. Also patients with previous history of surgery for trigeminal neuralgia were excluded.

\section{Imaging Protocol}

A 1.5 Tesla Siemens Magnetom MR machine was used for all studies. Routine sequences applied include T1, T2 and FLAIR axial sequences, coronal T2, sagittal T1/FLAIR and diffusion weighted sequence. A 3 dimensional constructive interference in steady state (3D CISS) sequence was acquired using the standard factors provided by the vendor. The acquisition matrix was $512{ }^{*} 512$ for a field of view of 230 * $230 \mathrm{~mm}$. Reconstruction in coronal and sagittal planes was done for interpretation of 3D CISS images.

All patients with space occupying lesions also underwent a 3D contrast enhanced fat suppressed T1- weighted imaging sequence.

\section{Image analysis}

All images were analysed and multiplanar reconstruction was done as required. Thinning of nerve, nerve indentation and change in signal intensity at the site of a vascular loop were taken as positive evidence of neurovascular compression. Patients with space occupying lesions underwent surgery and histopathological correlation was done for all these cases. 


\section{STATISTICAL ANALYSIS}

Statistical analysis was done using frequency analysis.

\section{RESULTS}

Out of 75 patients, 43 were men (58\%) and 32 were women (42\%). Mean age of study population was 52 years. Average age of males was 50.6 years and females 53.4 years. Abnormal magnetic resonance imaging findings were demonstrated in $57(76 \%)$ of the cases. The results are summarized in [Table/Fig-3].

Patients with neurovascular contact formed an overwhelming majority of the cases. $45(60 \%)$ out of 75 patients had a neurovascular contact demonstrable on the 3D CISS sequence. Neurovascular contact was not demonstrable in patients (20\%) had anterior inferior cerebellar artery as the cause of neurovascular contact [Table/Fig-4].

In all patients, neurovascular compression was seen on the same side as the patient symptoms. Asymptomatic neurovascular contact was found on the contralateral side in 3 out of 45 patients (6\%). Cerebellopontine angle lesions constituted $8 \%$ of the cases, with the demonstrated lesions being vestibular schwannoma (2 cases) [Table/Fig-5], trigeminal schwannoma (2 cases) [Table/Fig-6].

and one case each of epidermoid [Table/Fig-7] and arachnoid cyst [Table/Fig-8].

Three out of 75 patients (4\%), known cases of multiple sclerosis had developed symptoms of trigeminal neuralgia

\begin{tabular}{|l|l|c|c|c|}
\hline SL.NO & Etiology & Number of Males (\%) & Number of Females (\%) & Total (\%) \\
\hline \multirow{4}{*}{1} & Neurovascular Compression & $27(36)$ & $18(24)$ & $45(60)$ \\
\cline { 2 - 5 } & a. Superior cerebellar artery & $22(29.3)$ & $14(18.66)$ & $36(48)$ \\
\cline { 2 - 5 } & b. Anterior inferior cerebellar artery & $5(6.6)$ & $4(5.3)$ & $9(12)$ \\
\hline \multirow{4}{*}{2} & CP Angle Lesions & $4(5.3)$ & $2(2.6)$ & $6(8)$ \\
\cline { 2 - 5 } & a. Vestibular schwannoma & $2(2.6)$ & 0 & $2(2.6)$ \\
\cline { 2 - 5 } & b. Trigeminal schwannoma & $1(1.3)$ & $1(1.3)$ & $2(2.6)$ \\
\cline { 2 - 5 } & c. Epidermoid & 0 & $1(1.3)$ & $1(1.3)$ \\
\cline { 2 - 5 } & d. Arachnoid cyst & $1(1.3)$ & 0 & $1(1.3)$ \\
\hline \multirow{2}{*}{4} & Multiple sclerosis & $1(1.3)$ & $2(2.6)$ & $3(4)$ \\
\hline 5 & Infarcts & $2(2.6))$ & $1(1.3)$ & $3(4)$ \\
\hline \multirow{2}{*}{ NTable/Fig-3]: Table showing causes of trigeminal neuralgia in study population. } & $9(12)$ & $18(24)$ \\
\end{tabular}
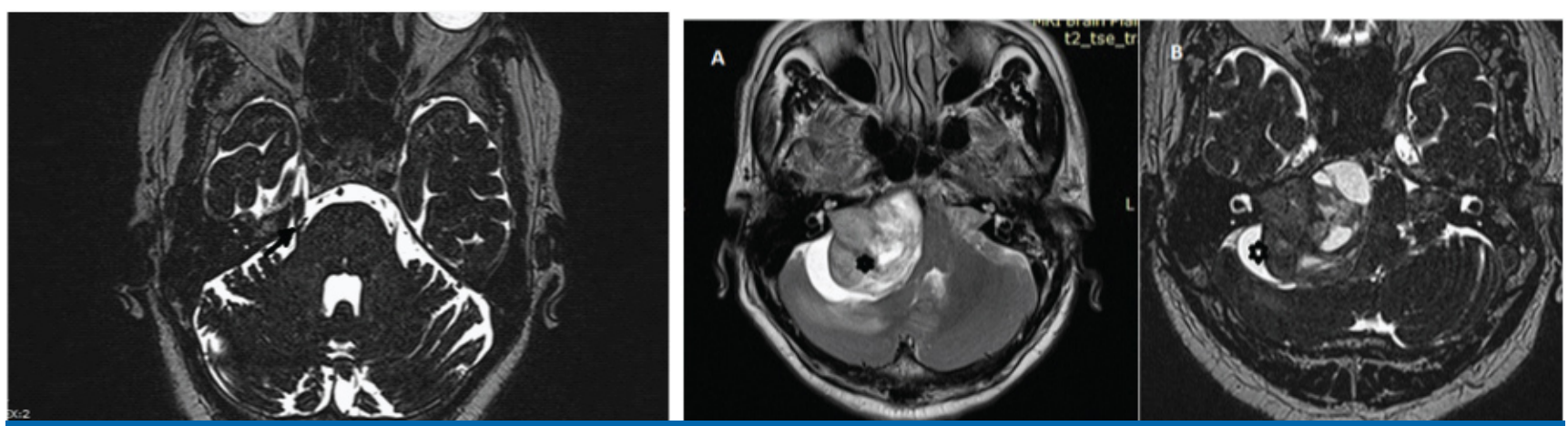

[Table/Fig-4]: Neurovascular compression. An axial CISS image shows right superior cerebellar artery indenting the trigeminal nerve in a patient with right hemifacial symptoms ( arrow).

[Table/Fig-5]: Vestibular schwannoma. A: Typical ice cream cone appearance of a vestibular schwannoma( $\left(^{\star}\right)$ on axial T2 image; B: The heterogenously hyperintense T2 lesion is also seen on axial CISS sequence.

other routine non-contrast sequences due to the limited spatial and contrast resolution. Few of the cases of neurovascular contact were also identifiable on contrast enhanced isotropic 3D gradient fat suppressed T1- weighted images.

36 out 45 patients (80\%) had superior cerebellar artery as the vessel in contact with the trigeminal nerve. 9 out of 45 over many months. No vascular loop was demonstrated. However, a demyelinating plaque was seen in the ipsilateral hemipons in both these patients and was assumed to be the cause of patients' symptoms [Table/Fig-9].

3 out of $75(4 \%)$ patients had chronic infarcts in the thalamus and hemipons on the same side as the patients' symptoms [Table/Fig-10]. 

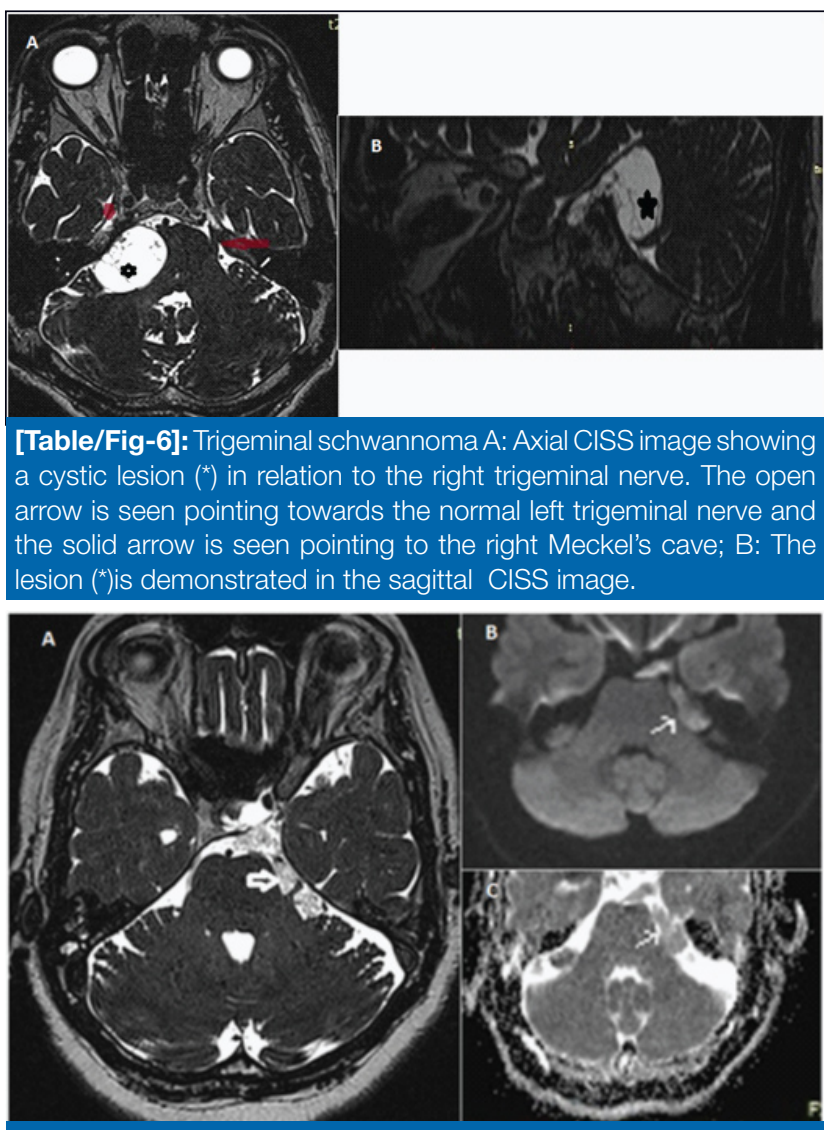

[Table/Fig-7]: Epidermoid. A: An irregular hyperintense lesion is seen surrounding the left trigeminal nerve (white arrow); B and C: Axial diffusion weighted image and corresponding ADC map demonstrating diffusion restriction in the lesion.

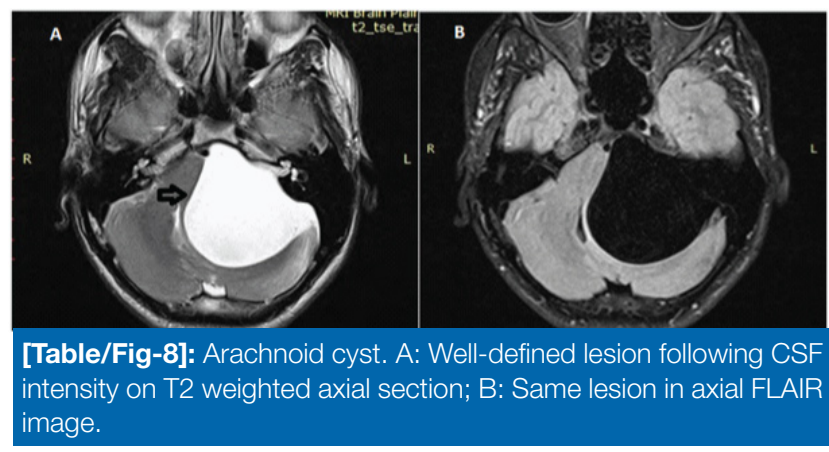

\section{DISCUSSION}

Our results have shown that there is a high percentage (76\%) of abnormal brain magnetic resonance imaging findings in patients with trigeminal neuralgia, with the causative lesions and their relative frequency correlating with other studies. A study on the frequency of abnormal brain magnetic resonance imaging findings in the Indian population has not been published previously.

CISS sequence had superior spatial and contrast resolution (due to the cisternographic effect) and was used in the demonstration of neurovascular contact. Trigeminal nerve was identified by tracing its course from the Meckel's case posteriorly to the pre-pontine cistern. The arteries were identified by tracing them to their origin from the basilar artery. The specific vessel was identified depending on the branching order of the basilar artery. In our study, 45 (60\%) patients, constituting $79 \%$ of abnormal brain MRI, had a demonstrable neurovascular contact on 3D CISS sequence, of which superior cerebellar artery was implicated in 80\% of the cases. This shows that 3D CISS sequence has the highest success in demonstrating positive findings on magnetic resonance imaging.

Jannetta was the first to recognize neurovascular compression as the cause of trigeminal neuralgia [12, 13]. Contrast enhanced magnetic resonance angiography can be used in addition to 3D CISS for demonstration and confirmation of the neurovascular contact [14]. Around $3-12 \%$ of asymptomatic patients had neurovascular contact on post- mortem studies emphasizing the need for interpreting imaging findings in the context of clinical symptoms [13].

Magnetic resonance imaging can also help characterize other lesions which present with symptoms of neurovascular compression, the next common group being cerebellopontine angle lesions. In our study, we had two cases of vestibular and trigeminal schwannoma and one case each of epidermoid cyst and arachnoid cyst. Schwannomas, also called neurilemmomas or neurinomas are benign tumors of neurogenic origin arising from perineural Schwann cells. Epidermoids are not actually neoplastic, originate from ectodermis and are composed of desquamated squamous epithelium. Other lesions occurring in the cerebellopontine region and causing trigeminal neuralgia include meningiomas, skull base tumors, etc. These posterior fossa lesions are believed to cause trigeminal neuralgia due to invasion/ encasement by the tumor itself, an interposed vessel or by distorting the posterior fossa and causing compression of trigeminal nerve against the skull base [12, 15, 16]. In some studies, the tumor was found to be on the contralateral side as the patients symptoms [17]. However, in our study, the patients symptoms and the lesion were found on the same side.

Multiple sclerosis is the most common demyelinating disease and is a leading cause of non- traumatic neurologic disability. It is also a well- recognized cause of trigeminal neuralgia. About $1-5 \%$ of patients with multiple sclerosis develop trigeminal neuralgia. In our study, three patients with multiple sclerosis had symptoms of trigeminal neuralgia. The cause is a demyelinating lesion affecting the pontine trigeminal pathways $[18,19]$.

Trigeminal neuralgia can also be seen in patients with brain stem lesions that cannot be categorized as multiple sclerosis-such as infarcts [20]. 

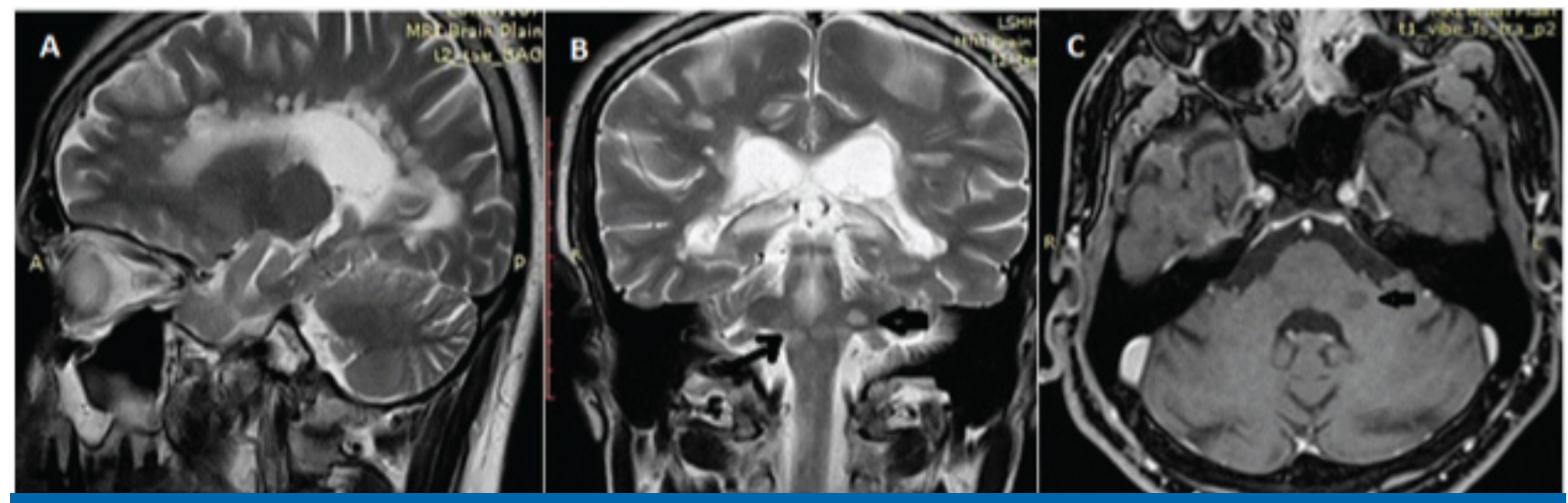

[Table/Fig-9]: Multiple sclerosis causing trigeminal neuralgia. A: Sagittal T2 weighted image showing circumscribed T2 hyperintense lesions at the callososeptal interface; B: Open arrow is pointing towards a demyelinating plaque in the left middle cerebellar peduncle. Another arrow is pointing towards multiple plaques in the brain stem; C: Axial contrast enhanced fat suppressed image showing the non-enhancing plaque in the left middle cerebellar peduncle.

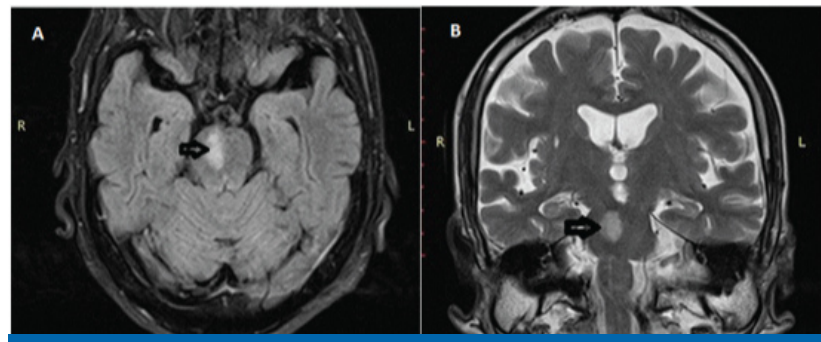

[Table/Fig-10]: Brain stem infarct A. Axial FLAIR image showing infarct in the right hemipons as a hyperintense area; B. The same lesion is seen in the coronal T2 weighted image as a hyperintense area.

Trigeminal neuralgia can be treated either medically or by surgical means. Carbamazepine is the drug of choice used in the treatment of trigeminal neuralgia. Surgical treatment options can be either microvascular decompression or ablative therapy. In addition to diagnosis, magnetic resonance imaging is highly useful in pre- operative planning and in determining prognosis [4].

\section{LIMITATIONS}

The limitations of the above study include small sample size, no post- surgical correlation of neurovascular compression, no comparison with post- contrast MR imaging and no postoperative follow-up of pain relief in patients who underwent surgery.

\section{CONCLUSION}

Abnormal findings on brain $\mathrm{MRI}$ are seen in a large number of patients with trigeminal neuralgia. Magnetic resonance imaging with 3D CISS sequence is considered the first line of investigation in patients with trigeminal neuralgia. However, in spite of its established usefulness in determining the cause of trigeminal neuralgia, there is a certain reluctance of practitioners in India in ordering MRI for investigating trigeminal neuralgia. $\mathrm{MRI}$ is usually requested for patients with refractory trigeminal neuralgia. It is likely that part of the reason for this is that $\mathrm{MRI}$ though available more than it was a few years ago, it is not as widespread as we'd like to believe. Other than neurovascular compression, MRI also has use in ruling out other causes of trigeminal neuropathy such as cerebellopontine angle lesions. Thus, magnetic resonance imaging helps in determining patients who will benefit from surgical treatment. This will go a long way in helping to reduce the morbidity in patients with trigeminal neuralgia.

\section{REFERENCES}

[1] Majoie CBLM, Verbeteen B, Dol JA, Peeters FLM. Trigeminal neuropathy: evaluation with MR imaging. Radiographics. 1995;15: 795-811.

[2] Woolfall P, Coulthard A. Trigeminal nerve: anatomy and pathology. BMJ. 2001;74:458-67.

[3] Headache Classification Committee of the International Headache Society $(\mathrm{IHS})$. The International Classification of Headache Disorders, $3^{\text {rd }}$ edition (beta version). Cephalagia. 2013;33:629-808.

[4] Bennetto L, Patel N K, Fuller G. Trigeminal neuralgia and it's management. BMJ. 2007;334; 201-05.

[5] Obermann M. Treatment options in trigeminal neuralgia. Ther Adv Neurol Disord. 2010; 3:107-15.

[6] Yoshino N, Akimoto H, Yamada H, Nagaoka T, Tetsumara A, Kurabayashi T et al. Trigeminal neuralgia: evaluation of neuralgic manifestation and site of neurovascular compression with $3 \mathrm{D}$ CISS MR imaging and MR angiography. Radiology. 2003; 228:539-45.

[7] Hingwala D, Chatterjee S, Kesavadas C, Thomas B, Kapilamoorthy TR. Applications of 3D CISS sequence for problem solving in neuroimaging. The Indian Journal of Radiology \& Imaging. 2011;2:90-97.

[8] Yousry I, Camelio S, Schmid UD, Horsfield MA, Wiesmann M, Bruckmann $\mathrm{H}$ et al. Visualization of cranial nerves I- XII: value of 3D CISS and T2- weighted FSE sequences. Eur Radiol. 2000;10:1061-67. 
[9] Lutz J, Linn J, Thon N, Stahl R, Seelos K, Mehrkens JH, et al. Trigeminal neuralgia due to neurovascular compression: high-spatial-resolution diffusion- tensor imaging reveals microstructural neural changes. Radiology. 2011;258:524-30.

[10] Harsha KJ. Imaging of vascular causes of trigeminal neuralgia. Journal of Neuroradiology. 2012;39:281- 89.

[11] Neetu S, Sunil K, Ashish A, Jayantee K, Usha Kant M. Microstructural abnormalities of the trigeminal nerve by diffusiontensor imaging in trigeminal neuralgia without neurovascular compression. Neuroradiol J. 2016;29(1):13-18.

[12] Love S, Coakham HB. Trigeminal neuralgia: pathology and pathogenesis. Brain. 2001;124:2347-60.

[13] Nurmikko TJ, Eldridge PR. Trigeminal neuralgia-pathophysiology, diagnosis and current treatment. $\mathrm{Br} J$ Anaesth. 2001;87:117-32.

[14] Patel NK, Aquilina K, Clarke Y, Renowden SA, Coakham HB. How accurate is magnetic resonance angiography in predicting neurovascular compression in patients with trigeminal

\section{AUTHOR(S):}

1. Dr. Vijaykumar Kenchanahalli Rangaswamy

2. Dr. Maskal Revanna Srinivas

3. Dr. Deepashri Basavalingu

4. Dr. Bangalore Rangaswamy Nagaraj

\section{PARTICULARS OF CONTRIBUTORS:}

1. Associate Professor, Department of Radio-Diagnosis, Bangalore Medical College and Research Institute, Bangalore, India.

2. Assistant Professor, Department of Radio-Diagnosis, Bangalore Medical College and Research Institute, Bangalore, India.

3. Post- Graduate Student, Department of RadioDiagnosis, Bangalore Medical College and Research Institute, Bangalore, India. neuralgia? A prospective, single-blinded comparative study. $\mathrm{Br}$ J Neurosurg.2003;17:60-64.

[15] Shulev Y, Trashin A, Gordienko K. Secondary trigeminal neuralgia in cerebellopontine angle tumors. Skull Base. 2011;21:287-94.

[16] Kobata H, Kondo A, Iwasaki K. Cerebellopontine angle epidermoids presenting with cranial nerve hyperactive dysfunction: pathogenesis and long-term surgical results in 30 patients. Neurosurgery. 2002;50:276-85.

[17] Larner A J. False localizing signs. I Neurol Neurosurg Psychiatry.2003;74: 415- 18.

[18] Mills RJ, Young CA, Smith ET. Central trigeminal involvement in multiple sclerosis using high-resolution MRI at $3 \mathrm{~T}$. The British Journal of Radiology. 2010;83:493-98.

[19] Cruccu G, Biasotta A, Di Rezza S, Fiorelli M, Galeotti F, Innocenti P. Trigeminal neuralgia and pain related to multiple sclerosis. Pain. 2009;143:186-91.

[20] Peker S, Akansel G, Sun I, Pamir NM. Trigeminal neuralgia due to pontine infarction. Headache. 2004;44:1043-45.

4. Professor and Head, Department of Radio-Diagnosis, Bangalore Medical College and Research Institute, Bangalore, India.

\section{NAME, ADDRESS, E-MAIL ID OF THE CORRESPONDING AUTHOR:}

Dr. Deepashri Basavalingu, $105,4^{\text {th }}$ Cross, First Stage Gangothri Layout, Mysore-570009, Karnataka, India.

E-mail: deepashri.b@gmail.com

FINANCIAL OR OTHER COMPETING INTERESTS: None. 